\title{
Macrophage depletion impairs neonatal tendon regeneration
}

Kristen L. Howell, Deepak A. Kaji, Angela Montero, Kenji Yeoh, Philip Nasser, Alice H. Huang

Department of Orthopaedics, Icahn School of Medicine at Mount Sinai, New York, NY 10029

\section{Corresponding Author:}

Alice Huang, $\mathrm{PhD}$

Associate Professor

Dept of Orthopaedics, Icahn School of Medicine at Mount Sinai

1 Gustave Levy PI, Box 1188

NY, NY 10029

Phone: (212) 241-1158

Email: alice.huang@mssm.edu

Date: $1 / 7 / 2021$

Keywords: tendon healing, macrophages, regeneration, mouse

Running Title: Macrophages are required for tendon regeneration 


\section{ABSTRACT}

48 Tendons are dense connective tissues that transmit muscle forces to the skeleton. After adult 49 injury, healing potential is generally poor and dominated by scar formation. Although the 50 immune response is a key feature of healing, the specific immune cells and signals that drive 51 tendon healing have not been fully defined. In particular, the immune regulators underlying 52 tendon regeneration are almost completely undetermined due to a paucity of tendon 53 regeneration models. Using a mouse model of neonatal tendon regeneration, we screened for 54 immune-related markers and identified upregulation of several genes associated with 55 inflammation, macrophage chemotaxis, and TGF $\beta$ signaling after injury. Depletion of 56 macrophages using AP20187 treatment of MaFIA mice resulted in impaired functional healing, 57 reduced cell proliferation, reduced ScxGFP+ neo-tendon formation, and altered tendon gene 58 expression. Collectively, these results show that inflammation is a key component of neonatal 59 tendon regeneration and demonstrate a requirement for macrophages in effective functional 60 healing. 


\section{INTRODUCTION}

Tendons are dense, connective tissues that connect muscle to the skeleton (1). Injury to tendons is extremely common and results in pain and sustained loss of mechanical function (25). This is largely due to the poor healing capacity of tendons and inability restore native matrix architecture since tendons heal by disorganized scar formation $(5,6)$. Currently, treatment options remain few and the gold standard of treatment is surgical repair, which does not regenerate tendon structure or function. Identifying novel biological mechanisms that improve functional tendon regeneration is therefore an important unmet need.

Toward that end, we previously established a neonatal mouse model of functional tendon regeneration after Achilles tendon transection without repair (7). We found that neonatal tendon regeneration was characterized by early proliferation of tendon cells, followed by their recruitment into the injury site to form a tenogenic neo-tendon. In the absence of tendon cell recruitment, functional properties were not restored (8). Using a similar injury model in adult mice, we also found that adult tendon healing was characterized by fibrotic scar formation, minimal tendon cell proliferation, and persistently reduced functional properties (7), consistent with studies from other groups (9-11).

Although the immune response is a key aspect of tendon healing, the specific immune cells and signals underlying tendon healing have yet to be fully defined. It is generally accepted that tendon healing is initiated by infiltration of pro-inflammatory macrophages (M1-like macrophages) followed by resolution of inflammation by alternatively activated macrophages (M2-like macrophages) (12-15). However, the role of these macrophage populations in promoting or inhibiting tendon healing is still unclear (16). While therapeutic strategies that enhance the presence of M2-like macrophages appear to promote functional tendon healing $(17,18)$, other studies suggest that increased M2-like macrophage polarization can also drive fibrotic scar formation (19). Most of these studies are correlative however and there are still very few studies that directly test the role of macrophages in tendon healing via ablation or depletion. One well-established method for macrophage depletion is delivery of liposomal clodronate (20). In adult mouse models of Achilles tendon transection and repair, macrophage depletion by clodronate resulted in reduced cell proliferation and improved mechanical properties $(21,22)$. Similarly, clodronate depletion in an ACL reconstruction rat model showed improved mechanical properties at the tendon-bone interface (23). Despite these results suggesting that macrophages impair adult functional healing, there is also evidence indicating that increased macrophage recruitment can also improve tensile properties after adult tendon injury (24). To date, the role of neonatal macrophages in tendon healing have not been investigated.

To address these open questions, we determined the immune response during neonatal tendon healing by screening immune-related gene expression and tested the requirement for macrophages using an established genetic model of macrophage depletion to inducibly ablate macrophages. We hypothesized that neonatal tendons would exhibit an anti-inflammatory immune response after injury and that macrophage depletion would impair functional regeneration.

\section{MATERIALS AND METHODS}

\section{Experimental procedures}


104

105

106

107

108

109

110

111

112

113

114

115

116

117

118

119

120

121

122

123

124

125

126

127

128

129

130

131

132

133

134

135

136

137

138

139

140

141

142

143

144

145

146

147

148

149

For experiments, the transgenic Macrophage-Fas-Induced Apoptosis (MaFIA) mouse line (25) and ScxGFP tendon reporter line (26) were used. Although the MaFIA line allows GFPdetection of Csfr1-expressing monocytes and macrophages, we were only able detect MaFIAGFP by flow cytometry and not by fluorescence microscopy of either spleen or tendon. Flow cytometry was therefore used to quantify macrophage depletion in mice containing only the MaFIA allele. For other experiments, ScxGFP was incorporated into the MaFIA background to enable ScxGFP cell visualization with macrophage depletion (MaFIA/ScxGFP). EdU was given at $0.05 \mathrm{mg} 2$ hours prior to harvest to label proliferating cells. Global macrophage depletion was carried out using the homodimerizer AP20187 at $10 \mathrm{mg} / \mathrm{kg}$ in carrier solution by subcutaneous injection (Cat. \# 635058, Clontech) with carrier-treated controls (4\% EtOH, 10\% PEG-400, 1.7\% Tween20) injected in parallel. Littermates from the same litter were evenly split between AP20187-treatment or carrier-treatment. Full Achilles tendon transection without repair was carried out in neonates at P5, with male and female mice distributed evenly between groups. All procedures were approved by the Institutional Animal Care and Use Committee at Mount Sinai.

\section{Flow cytometry quantification}

Single cell suspensions were generated from Achilles tendons of MaFIA mice by enzymatic digestion with a solution containing $1 \mathrm{mg} / \mathrm{mL}$ collagenase I (Cat. \# LS004196, Worthington Biochemical) and $5 \mathrm{mg} / \mathrm{mL}$ collagenase IV (Cat. \# LS004188, Worthington Biochemical) for 2.5 hrs at 37C. Cells were then stained 1:1000 with DAPI (Cat. \# D1306, ThermoFisher) in 2\% FBS in PBS to detect live cells. Flow cytometry was carried out on an LSRIIA machine (BD Sciences) using FACSDiva software and analyses using FCS Express 7. Gating for MaFIAGFP was performed using control cells isolated in parallel from a wild type mouse with no GFP.

\section{Immunofluorescence, EdU detection, and fluorescence microscopy}

Limbs were immediately fixed in $4 \%$ PFA after harvest for 24 hours at $4^{\circ} \mathrm{C}$ and then decalcified in $50 \mathrm{mM}$ EDTA for $1-2$ weeks at $4^{\circ} \mathrm{C}$. To embed, limbs were incubated in $5 \%$ sucrose (1 hour) and $30 \%$ sucrose (overnight) at $4^{\circ} \mathrm{C}$ and then embedded in OCT medium (Cat. \# 23-730, Fisher Scientific). Transverse cryosections $(12 \mu \mathrm{m})$ were collected in alternating slides. Immunostaining for macrophages was carried out using an antibody against the global macrophage marker F4/80 (Cat. \# 14-4801, Affymetrix) and secondary detection by Cy5 (Cat. \# 712-175-150, Jackson ImmunoResearch). EdU labeling was detected with the Click it EdU kit in accordance with manufacturer's instructions (Cat. \# C10340, Life Technologies). Fluorescence images of EdU and ScxGFP were acquired using the Zeiss Axio Imager with optical sectioning by Apotome. Cell quantification was performed in Image $\mathrm{J}$ software using the CellCounter plugin. All images for quantifications were taken at the same exposure and image manipulations applied equally across samples.

RNA isolation, reverse transcription, and qRT-PCR

RNA was extracted from Achilles tendons using Trizol/chloroform. cDNA was synthesized by reverse transcription using the SuperScript VILO master mix (Cat. \# 11755050, Invitrogen). qPCR was carried out by Mount Sinai's core facilities using SYBR PCR Master Mix (Cat. \# 4309155, Thermo Fisher) and gene expression calculated using the $2^{-\Delta \Delta \mathrm{Ct}}$ method relative to Gapdh and carrier-treated control tendons at D3. Primers for I/1 $\beta$ (FWD: AGTTGACGGACCCCAAAAGAT; REV: GTTGATGTGCTGCTGCGAGA) and $1 / 10$ (FWD: ATTTGAATTCCCTGGGTGAGAAG; REV: CACAGGGGAGAAATCGATGACA) were used. Tendon gene primers were previously described (7).

\section{Gait analysis}


On D28, mice were gaited at $10 \mathrm{~cm} / \mathrm{s}$ for $3-4 \mathrm{~s}$ using the DigiGait Imaging System (Mouse Specifics Inc.). A high-speed digital camera was used to capture forelimb paw positions and parameters previously validated for mouse Achilles tendon injury (Swing, Brake, and Propel) were then calculated (7). All parameters were normalized to Stride length to account for differences in animal size since male and female mice were used.

\section{Biomechanical testing}

For biomechanical testing, Achilles tendons were dissected at D56, wrapped in PBS-soaked gauze and frozen at $-20 \mathrm{C}$ until time of testing. Tensile testing was performed in PBS at room temperature using custom 3D printed grips to secure the calcaneus bone and Achilles tendon $(27,28)$. Tendons were preloaded to $0.05 \mathrm{~N}$ for $\sim 1 \mathrm{~min}$ followed by ramp to failure at $1 \% / \mathrm{s}$.

\section{Statistical analysis}

Quantitative results are presented as mean \pm standard deviation. For tendon macrophage flow cytometry, functional properties, and gene expression, two way ANOVA was used for comparisons with independent variables of treatment (carrier vs AP20187) or injury; where significance was detected, posthoc testing was then carried out (Graphpad Prism). Flow cytometry of spleen macrophages and all other quantitative analyses were analyzed using Students t-tests. Significant outliers were detected using Grubb's test (Graphpad Prism). Significance was determined at $p<0.05$.

\section{RESULTS}

Neonatal tendon injury activates an early pro-inflammatory immune response

To define the immune response associated with neonatal tendon regeneration, we carried out a gene expression screen using the Taqman Array Mouse Immune Response panel comprising 96 genes. Neonatal injured and contralateral uninjured Achilles tendons were screened at 3 days post-injury (D3), prior to recruitment of tenogenic cells in the gap space. While we initially expected neonatal injured tendons to exhibit a minimal immune response, we found 27 genes were upregulated after injury ( $\geq 2$-fold, $p<0.05$, Table 1) with 4 genes were downregulated ( $\leq-2$ fold, $\mathrm{p}<0.05$, Table 2). Upregulated markers included genes associated with macrophages and monocyte/macrophage chemotaxis (Ccl2, Ccl3, Ccr2, Ccr7, Cd68) and T-cells (Tbx21, Cd3e, Ctla4, Cxcr3). Surprisingly, there appeared to be a robust inflammatory response as we detected upregulation of several pro-inflammatory cytokines $(I / 6, I / 1 \beta, I / 12 \beta, \| 18)$; however, at this timepoint the anti-inflammatory cytokine $/ / 10$ was also upregulated. In addition to upregulation of $\operatorname{Tgf} \beta 1$, we detected significant downregulation of $S k i$, which is a known negative regulator of TGF $\beta$ signaling. Collectively, our qPCR screen indicates that neonates mount a robust pro-inflammatory immune response after injury that may be driven by recruitment of macrophages and T-cells. 
Table 1: Differentially expressed genes detected by Taqman Array Mouse Immune Response panel in response to neonatal tendon injury at D3.

\begin{tabular}{|c|c|c|}
\hline Gene & Fold Change (Up-regulated) & p value \\
\hline Gzmb & 183.2675 & 0.045003 \\
\hline Ccl5 & 38.95 & 0.000215 \\
\hline $1 / 6$ & 23.5654 & 0.026617 \\
\hline Prf1 & 23.4663 & 0.006136 \\
\hline$T b \times 21$ & 22.367 & 0.003557 \\
\hline Sele & 18.1162 & 0.028513 \\
\hline Tnfrsf18 & 14.0241 & 0.009632 \\
\hline Cd3e & 13.907 & 0.006046 \\
\hline Ctla4 & 13.7629 & 0.00171 \\
\hline Vcam1 & 13.5854 & 0.013771 \\
\hline$/ / 1 \beta$ & 11.77 & 0.000642 \\
\hline $1 / 10$ & 11.3756 & 0.002664 \\
\hline $\mathrm{Ccl3}$ & 10.1302 & 0.017634 \\
\hline Ccl2 & 10.0745 & 0.024852 \\
\hline Ccr7 & 9.366 & 0.000269 \\
\hline Fasl & 7.1758 & 0.001069 \\
\hline$I / 12 \beta$ & 6.952 & 0.02003 \\
\hline H2-Eb1 & 6.9387 & 0.000538 \\
\hline Cd80 & 5.7704 & 0.030404 \\
\hline Ccr2 & 5.7292 & 0.005281 \\
\hline Cxcr3 & 4.0058 & 0.010695 \\
\hline Csf2 & 3.737 & 0.009016 \\
\hline Stat1 & 3.5504 & 0.040912 \\
\hline $1 / 18$ & 3.2386 & 0.045934 \\
\hline Cd68 & 3.1542 & 0.003324 \\
\hline Hmox1 & 2.4951 & 0.011452 \\
\hline$T g f b 1$ & 2.1161 & 0.011681 \\
\hline Genes & $\begin{array}{l}\text { Fold Change (Down- } \\
\text { regulated) }\end{array}$ & p value \\
\hline Nos2 & -2.3753 & 0.005565 \\
\hline Ski & -2.23 & 0.000631 \\
\hline Vegfa & -2.1495 & 0.009757 \\
\hline Ace & -2.0735 & 0.040496 \\
\hline
\end{tabular}


Increased macrophages and macrophage localization with tenocytes after neonatal tendon 192 injury

193 Analysis of the pan-macrophage marker F4/80 in transverse cryosections from uninjured tendon 194 (P8 post-birth) showed tendon-resident macrophages are normally sparsely detected in the 195 tendon periphery as well as in fascia surrounding the tendon (Figure 1A, B). By D3 post-injury, 196 we observed a dramatic increase in $\mathrm{F} 4 / 80+$ cells surrounding the injured tendon. Comparison of sections taken from the tendon stub far from the cut site as well as sections close to the cut site showed that $\mathrm{F} 4 / 80$ + macrophages were only located in close proximity to ScxGFP+ tendon cells 200
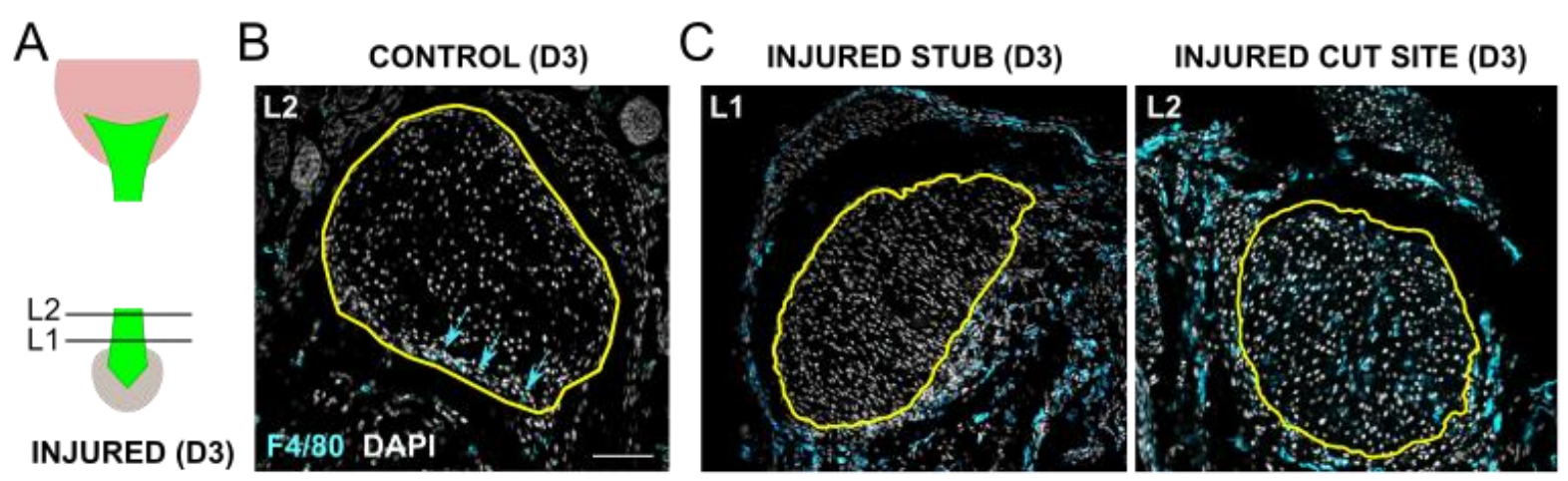

201

202

203

204

205

206

207

208

Figure 1: Macrophages are recruited after neonatal tendon injury and localize to cut site tendon cells. (A) Transverse cryosections were collected from tendon stub (L1) and cut site (L2) levels shown in schematic. Immunostaining for pan-macrophage marker F4/80 of (B) uninjured control and (C) injured tendon at D3 post-injury. Yellow outline indicates tendon region. Blue arrows point to sparse $\mathrm{F} 4 / 80+$ tendon-resident macrophages residing in tendon periphery. Scale bar: $100 \mu \mathrm{m}$. 
Repeated depletion of macrophages during postnatal growth resulted in adverse systemic effects unrelated to tendon healing

211 The close proximity of macrophages to tenocytes suggested potential interactions between these cells. We therefore tested the functional role of macrophages on neonatal tendon regeneration using the Macrophage Fas-Induced Apoptosis transgenic mutant (MaFIA) in which monocytes and macrophages express a mutant human FK506 binding protein. Macrophages could therefore be inducibly and reversibly depleted by delivery of the homodimerizer AP20187. For all experiments, AP20187 or carrier was injected for three consecutive days (P2, P3, P4) prior to Achilles tendon transection at P5 to deplete monocytes and macrophages prior to injury. We then tested three different regimens of macrophage depletion after P5 injury, including twice a week, once a week, or a single booster dose at d7 post-injury. When AP20187 was delivered twice a week following injury, we observed swelling of both injured and uninjured hindlimbs of treated mice and impaired growth (Figure 2A-C). Once a week treatment improved survival of mice out to nearly d56, however restricted growth was observed beginning at d35 (Figure 2DF). Swelling was also observed in both injured and uninjured hindlimbs of surviving mice at d56. Finally, we treated mice with a single booster injection at $\mathrm{d} 7$ and observed no difference in growth and no swelling of hindlimbs (Figure 3A-C). Since the single booster treatment showed minimal systemic effects and has also been reported in other studies (29), we carried out subsequent analyses using this treatment regimen. Analysis of ablation efficiency by flow cytometry of MaFIA $\mathrm{GFP}^{\mathrm{H}}$ showed $86 \%$ reduction in macrophages at d3 in the injured tendon and 93\% reduction in the spleen following AP20187 treatment (Figure 3D). 


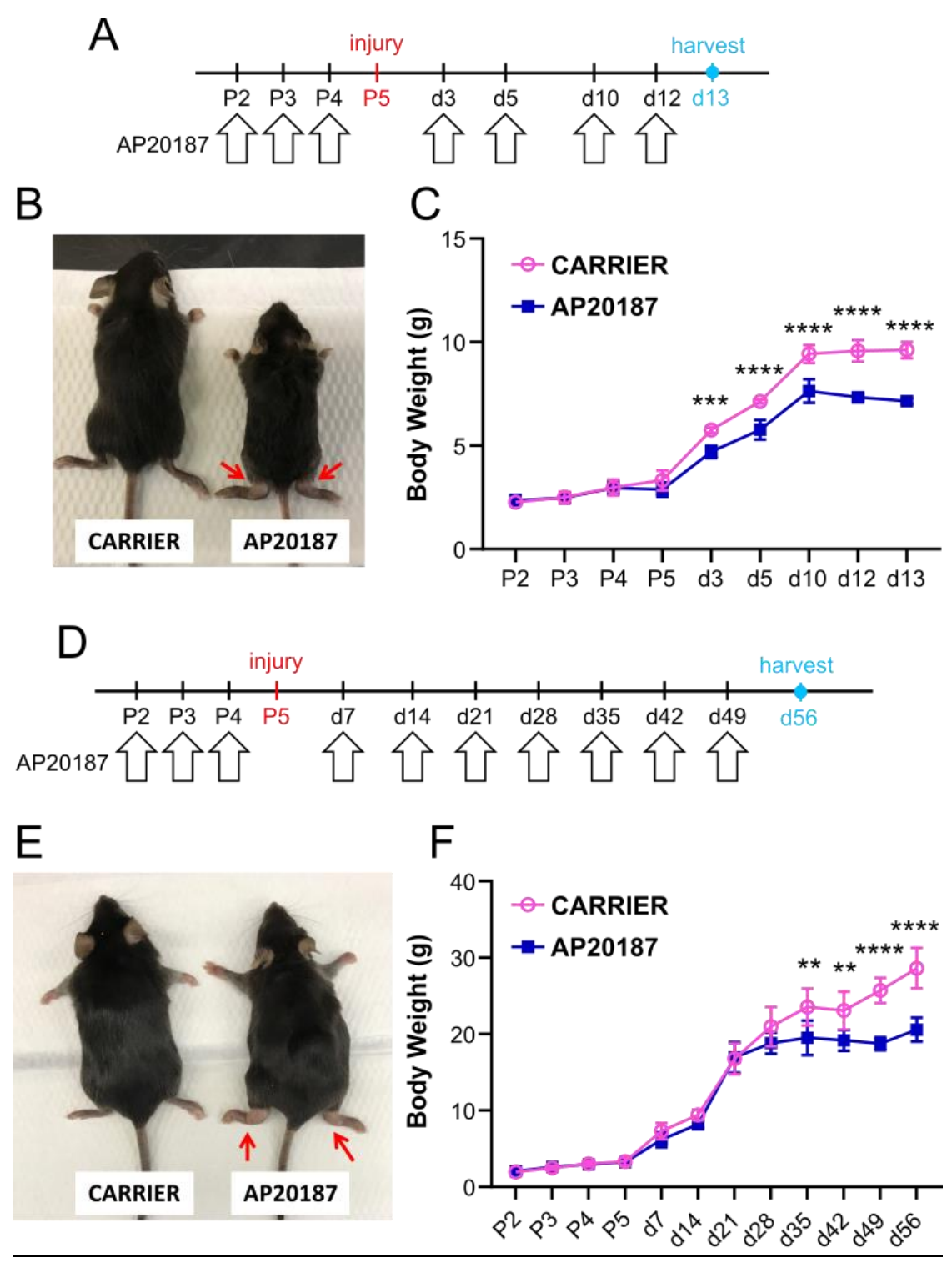

Figure 2: Prolonged and repeated depletion of macrophages results in adverse systemic effects. (A) Schematic showing twice a week dosing regimen of AP20187 after P5 Achilles tendon injury. (B) AP20187-treated mice were smaller and showed abnormal swelling of ankles at D13 (red arrows) with (C) impaired weight gain ( $n=3-5)$. (D) Schematic showing once a week dosing regimen of AP20187 after P5 Achilles tendon injury. (E) AP20187-treated mice also developed abnormal swelling of ankles at D56 (red arrows) and (F) impaired weight gain at later timepoints $(n=3-5) .{ }^{* *} p<0.01 ;{ }^{* * *} p<0.001{ }^{* * * *} p<0.0001$. 
A

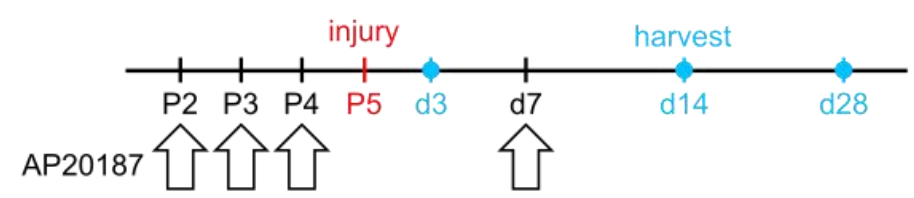

B

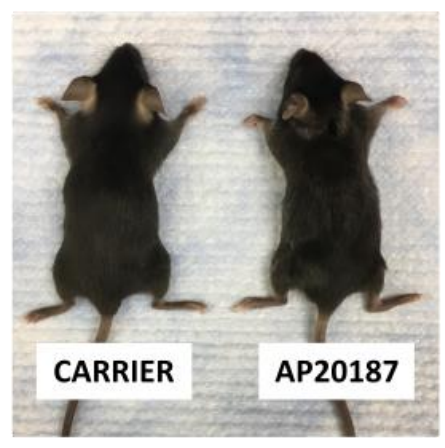

C
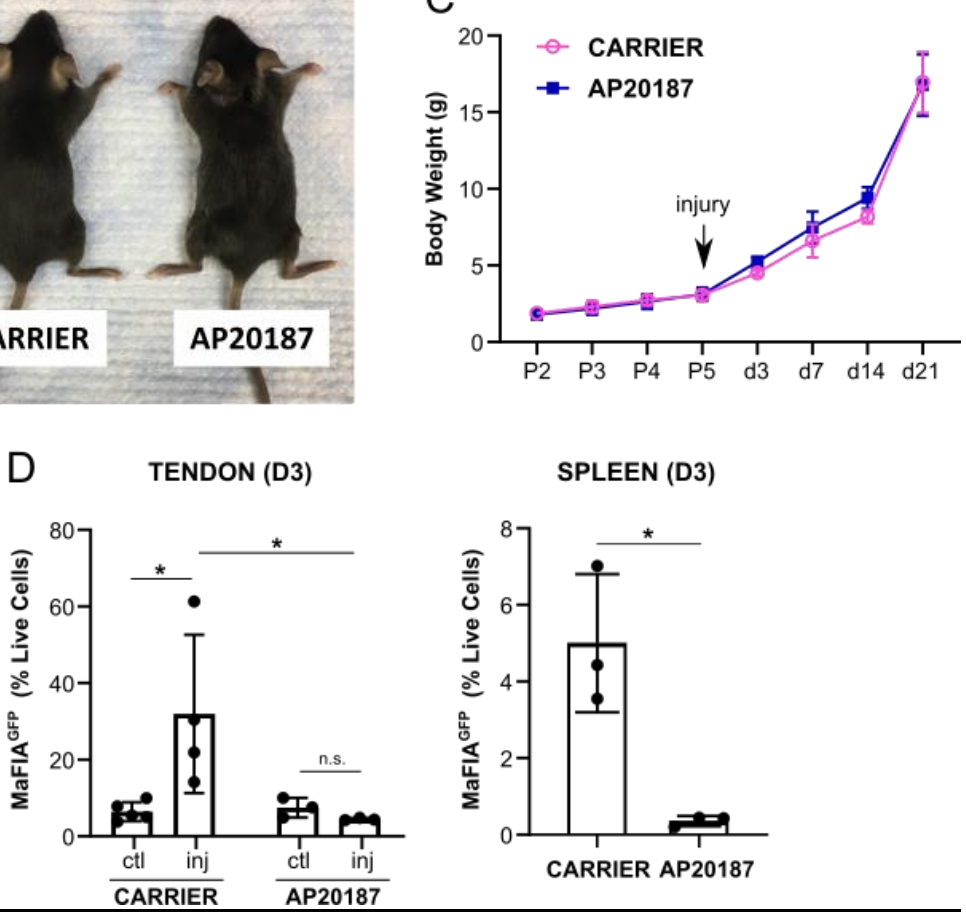

Figure 3: Validation of macrophage depletion by AP20187. (A) Schematic showing a single booster dose of AP20187 at D7 after P5 Achilles tendon injury. (B) AP20187-treated mice did not demonstrate swelling of hindlimbs compared to carrier-treated at D28. (C) Weight gain was comparable at all timepoints $(n=4-9)$. (D) Flow cytometry quantification of MaFIA GFP cells at D3 in tendon and spleen. ${ }^{*} p<0.05$. 
To determine functional healing when macrophages were ablated, we carried out gait analysis and tensile testing. In previous studies, we identified swing, brake, and propel parameters (normalized to stride length) were affected by injury $(7,8)$. Analysis of gait at 28 showed no differences with AP20187-treatment, either compared to contralateral uninjured control or compared to carrier treatment for any of the three parameters (Figure 4). However, direct tensile testing of tendons at D56 showed impaired functional restoration with macrophage ablation after injury. Stiffness was reduced in AP20187-treated injured tendons compared to the contralateral control tendon and max force was reduced compared to both contralateral control and carrier injured tendons (Figure 5). There was no difference in mechanical properties between control and injured limbs with carrier treatment. These results indicate that macrophages are required for functional neonatal tendon healing.
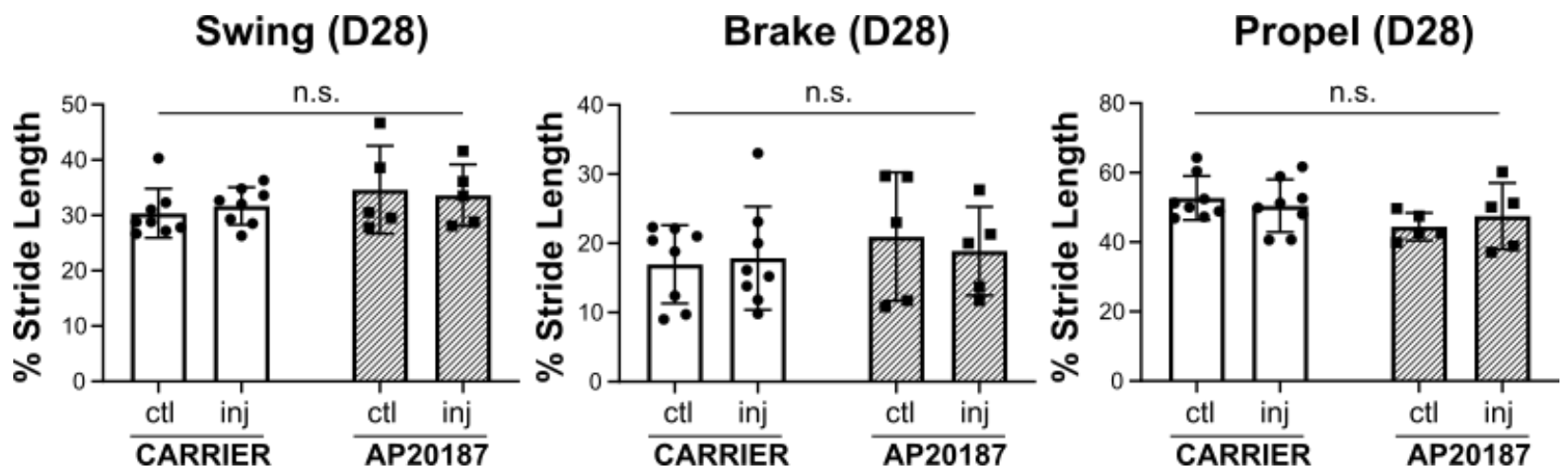

Figure 4: Recovery of gait after tendon injury was not affected by macrophage depletion. Gait analysis showed functional recovery of all gait parameters (Swing, Brake, and Propel) at D28 post-injury regardless of treatment. n.s. indicates $p>0.05$. $n=5-8$.

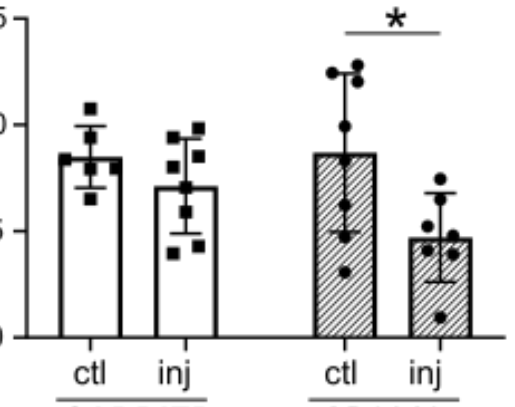

CARRIER

AP20187

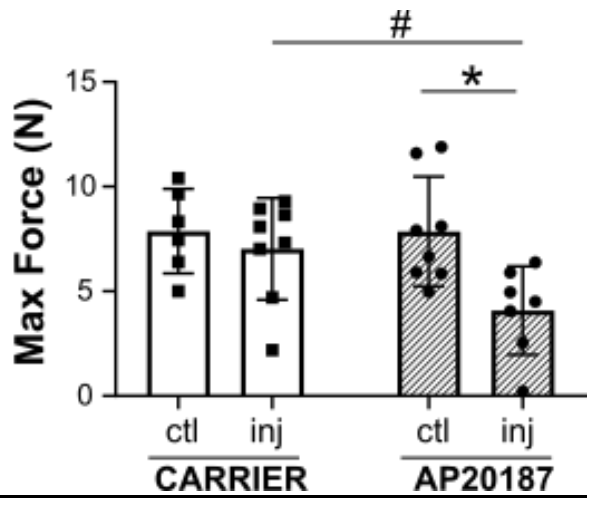

Figure 5: Mechanical properties are impaired after tendon injury with macrophage depletion. Tensile testing of Achilles tendons at D56 post-injury showed AP20187-treated tendons did not fully restore stiffness and max force after injury compared to contralateral controls $(n=6-8)$. ${ }^{*} p<0.05 \# p<0.1$. 
271 Early ScxGFP+ cell proliferation and subsequent ScxGFP+ neo-tendon formation is impaired 272 with macrophage depletion

273 To understand the cellular deficits that might be associated with functional loss, we analyzed 274 proliferation at D3 by EdU incorporation (2 hrs) and ScxGFP neo-tendon formation at D14 275 (Figure 6A). With carrier treatment, intense proliferation of both ScxGFP+ and ScxGFP-cells 276 was observed at D3 in the tendon cut site (Figure 6B). In contrast, minimal proliferation 277 occurred with AP20187 treatment and the number of proliferating ScxGFP+ cells was reduced. 278 By D14, a robust ScxGFP+ neo-tendon was formed in carrier-treated mice, however 279 macrophage depletion resulted in significantly reduced ScxGFP+ area (Figure 6C). Taken 280 together, this suggests that macrophage depletion resulted in defects in tendon cell proliferation 281 leading to reduced ScxGFP cell recruitment and neo-tendon formation. 

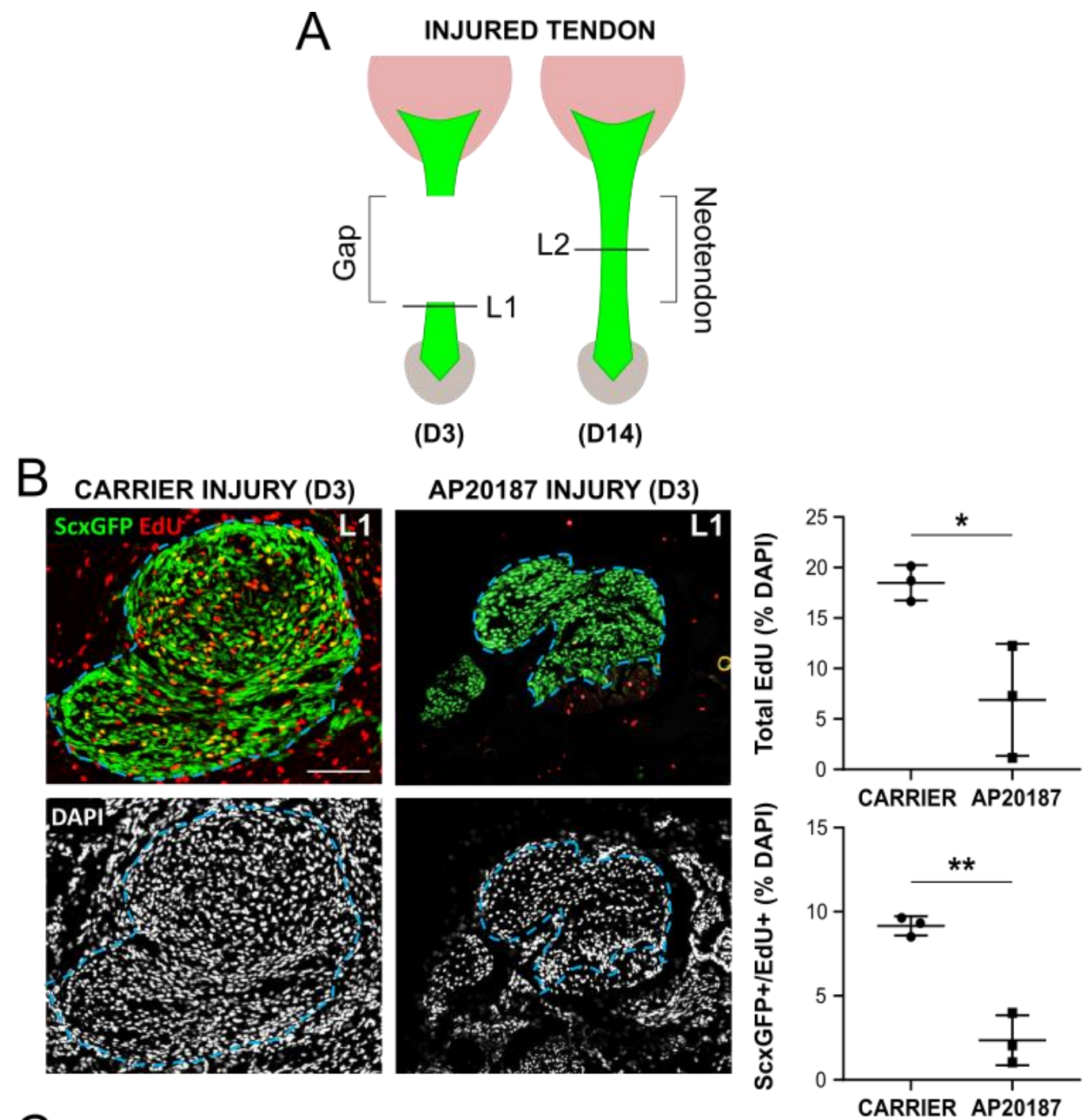

C

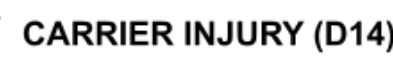

\section{SCXGFP}

AP20187 INJURY (D14)
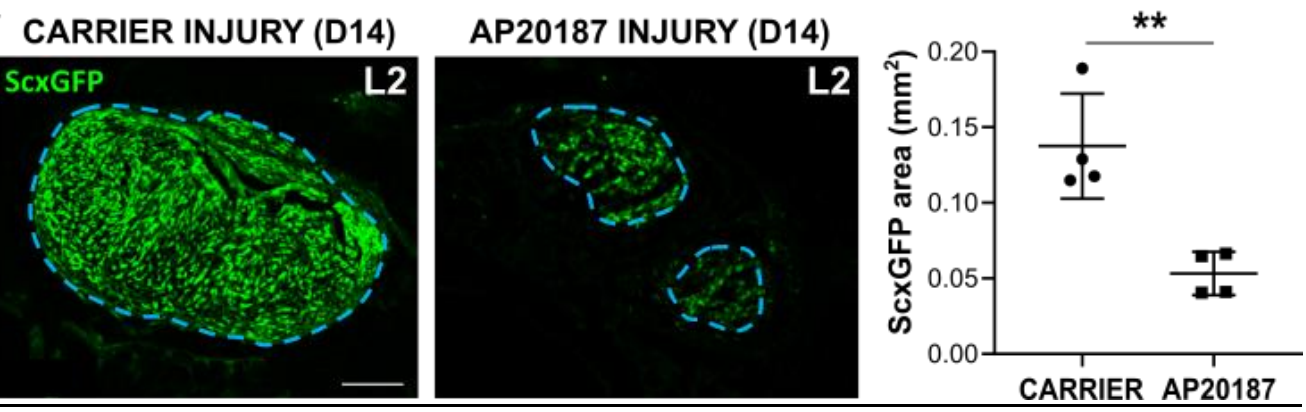

Figure 6: Reduced proliferation and ScxGFP neo-tendon formation after tendon injury with macrophage depletion. (A) Transverse cryosections were collected from tendon cut site at D3 (L1) and neo-tendon region at D14 (L2). (B) EdU and ScxGFP imaging and cell quantification at D3 post-injury $(n=3)$. (C) ScxGFP area quantification at D14 post-injury $(n=4)$. Blue dashed outlines show tendon region of interest based on ScxGFP expression. * $p<0.05$ ** $289 \quad \mathrm{p}<0.01$. 
Macrophage depletion results in altered gene expression of the contralateral control tendon

Although the neo-tendon area was reduced with macrophage depletion, we still observed a population of ScxGFP+ cells recruited at D14, indicating a positive tendon phenotype. To better define the tenogenic phenotype of these cells, we carried out gene expression analysis at D3 and D14 by qPCR. Expression of known markers Scx, Tnmd, and Mkx was reduced in carrier treated injured limbs relative to contralateral controls at D3 (Figure 7A), consistent with our prior studies (8). In AP20187-treated limbs, injury induced a less predictable response. While Tnmd expression decreased with injury, Scx and Mkx levels were not significantly different $(p>0.1)$. At D14, a small reduction in Tnmd and Mkx expression was still observed in carrier-treated injured limb relative to control with no difference in AP20187-treated limbs (Figure 7B). However, this was due to a decrease in Tnmd and Mkx in AP20187-treated control limbs (compared to carriertreated controls). Unexpectedly, Scx expression in AP20187-treated control limbs was decreased at D3 relative to carrier-treated control limbs, but dramatically increased by D14 relative to all other groups (Figure 7A, B). This may indicate a potential rebounding effect, although ScxGFP expression was not visibly altered (not shown). There was no difference in tendon gene expression between carrier-treated and AP-treated injured limbs at any timepoint.
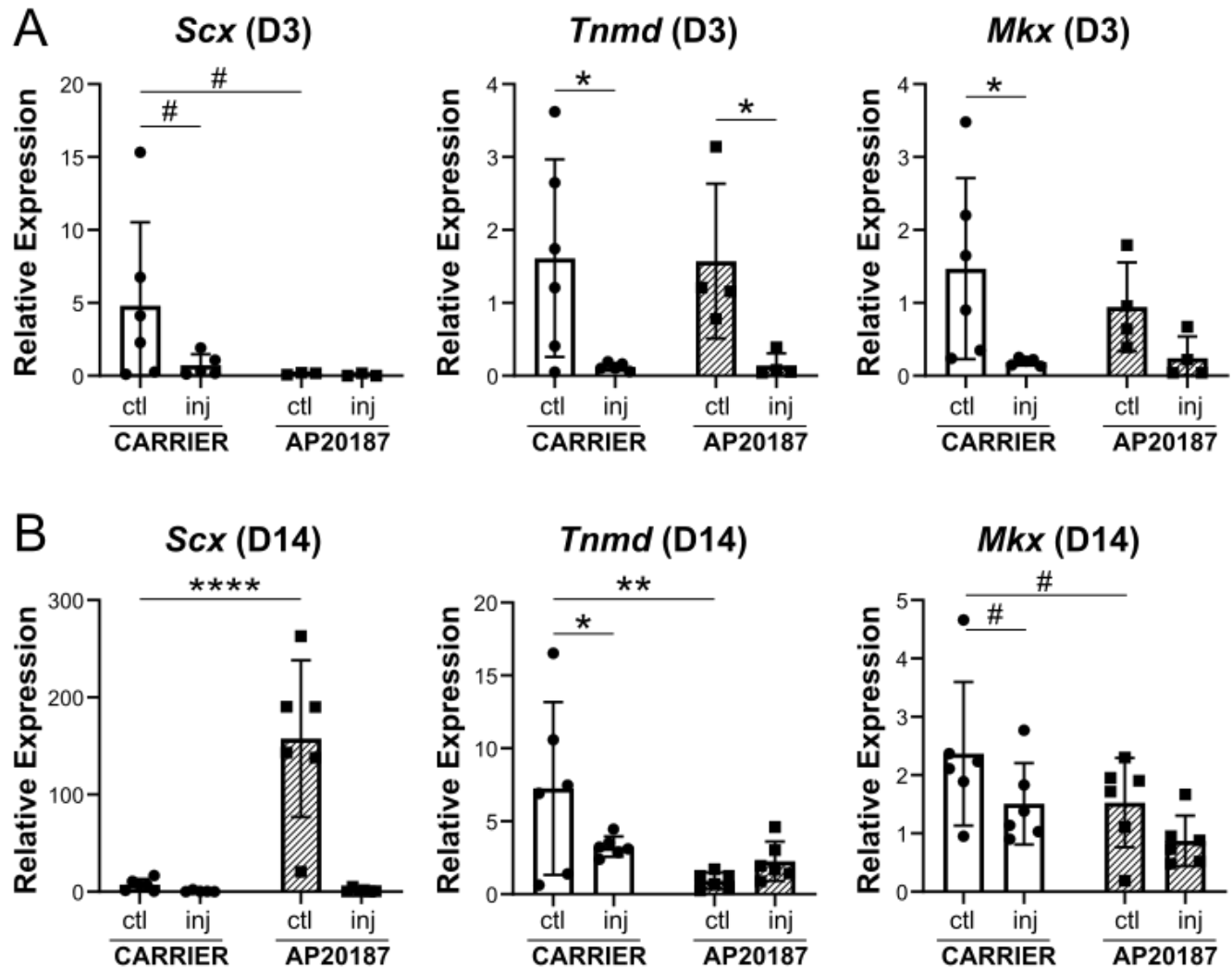

Figure 7: Tendon gene expression is altered with macrophage depletion. Expression of tendon genes Scx, Tnmd, and Mkx at (A) D3 and (B) D14 post-injury $(n=4-6) .{ }^{*} p<0.05$ ** $p<0.01^{* * * *} p<0.0001 \# p<0.1$. 
314 To determine the effects of macrophage depletion on local inflammation, $/ / 1 \beta$ and $/ / 10$ expression was determined by qPCR at D3 and D14. At D3, II1 $\beta$ expression was increased with injury compared to controls, regardless of treatment (Figure $\mathbf{8 A}$ ). Levels of $I 11 \beta$ was higher in AP20187-treated injured limbs relative to carrier-limbs, although the difference did not reach significance $(p=0.08)$ due to high variability. At $D 14$, there was persistent $I / 1 \beta$ upregulation in carrier-treated injured limbs, but no difference with AP20187-treatment (Figure 8B). There was no difference in $/ / 10$ expression at any timepoint for either treatment or injury. Overall, this data suggests that there may be somewhat early elevation of inflammation with macrophage depletion.
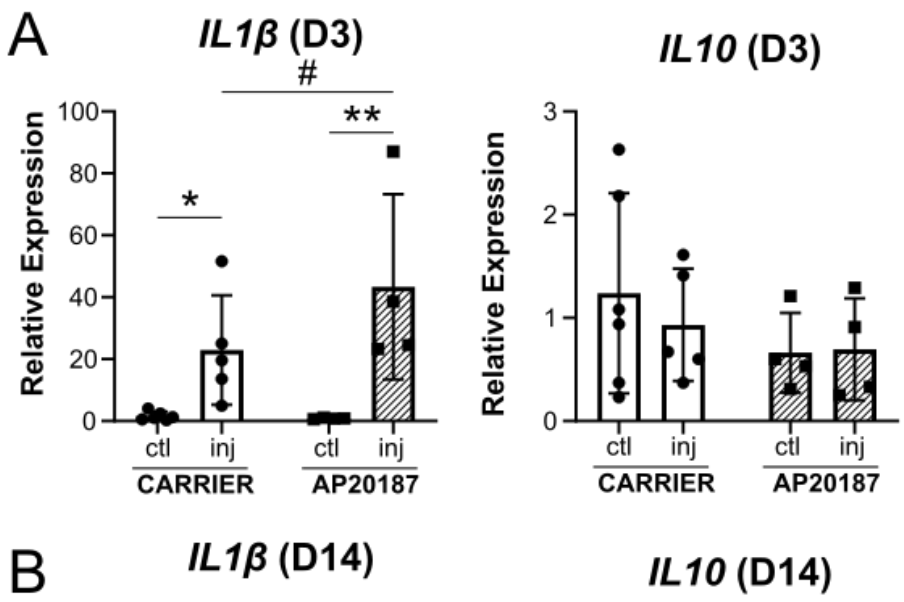

\section{IL10 (D14)}
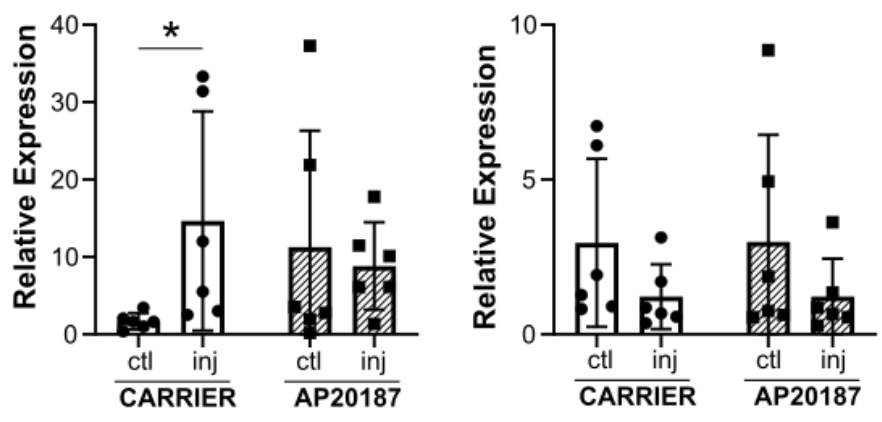

Figure 8: Expression of cytokines with macrophage depletion. Expression of $/ / 1 \beta$ and $/ / 10$ at (A) D3 and (B) D14 post-injury $(n=4-6)$. ${ }^{*} p<0.05{ }^{* *} p<0.01 \# p<0.1$. 


\section{DISCUSSION}

In this study, we screened for immune gene expression markers after tendon injury and found upregulation of several pro-inflammatory markers, suggesting that early inflammation is a key feature of neonatal tendon regeneration. This was contrary to our initial hypothesis, as it was previously suggested that scar-less fetal regeneration occurred in a permissive immune environment characterized by minimal inflammation (30-32). Increased inflammation in the fetal environment resulted in failed regeneration of fetal skin and tendons $(30,33)$; this could be rescued by dampening inflammation via IL-10 over-expression (30). Interestingly, there is also evidence that adult tissues are unable to regenerate when placed in the fetal environment, suggesting that a permissive immune environment may be necessary but not sufficient to fully transform adult scar-mediated healing (34). Although fetal tendons transplanted into the adult environment were still able to regenerate (35), immunodeficient hosts were used in these studies and the fetal tendons therefore did not experience a normal adult immune response to injury. To date, there are still few studies focused on the immune regulation of neonatal regeneration. However, it is well appreciated that the neonatal immune system is less mature compared to adult and is biased toward type 2 immunity in response to infections (36, 37). Many categories of neonatal immune cells (including neutrophils, natural killer cells, monocytes, and others) also show substantial functional differences and numbers compared to adults (37), reflecting the unique immune environment of the neonate. While we observed enhanced expression of pro-inflammatory markers, analyses were limited to a single early timepoint. Ongoing studies will define the temporal dynamics of inflammation and its resolution (or persistence) in neonates in direct comparison with adult counterparts after tendon injury. It may be that neonatal inflammation is reduced compared to adults or is more rapidly resolved.

Depletion of macrophages during early healing resulted in impaired functional properties likely due to reduced ScxGFP tendon cell proliferation, recruitment, and neo-tendon formation. The close proximity of macrophages to tendon cells at D3 suggests that secreted factors from macrophages may stimulate tendon cell proliferation. While TGF $\beta$ is an attractive candidate based on our gene expression array screen and has been implicated in other macrophage depletion models of tendon healing $(21,23)$ as well as fetal tendon healing $(35,38)$, our previous studies inhibiting TGF $\beta$ signaling showed no effect on tendon cell proliferation at D3 (8). The molecular regulators for early cell proliferation is therefore still unknown. These factors may be identified by transcriptional profiling by RNA sequencing of isolated MaFIAGFP macrophages or by single cell RNA sequencing.

One major limitation of this study includes the timing of AP20187 delivery and macrophage depletion. The goal of this study was to determine broadly the role of macrophages; we therefore depleted macrophages prior to injury with an additional booster injection at D7. While previous research in adult tendons suggest that M2-like macrophages do not emerge until D28 in the healing timeline (12), it is possible we also depleted M2-like macrophages. In our study, we observed reduced ScxGFP+ cell proliferation at D3, during the time when M1-like macrophages are expected to be dominant. This suggests that M1-like macrophages are important for stimulating tendon cell proliferation. While other studies also showed a requirement for macrophages in cell proliferation $(21,22)$, this is the first study to distinguish tendon cells (ScxGFP+) from exogenous or dedifferentiated (ScxGFP-) cells. Our finding that inflammation may be crucial for the early healing response is consistent with other research showing that inhibition of inflammation too early in tendon healing can have detrimental outcomes on functional properties (39). Future studies will determine the temporal dynamics and functions of distinct macrophage sub-populations recruited during neonatal tendon healing and test depletion strategies targeting early (D0-D3), middle (D5-D9), and late (D20-24) phases. Temporal depletion of macrophages will also determine whether impaired neo-tendon formation 
at D14 is due solely to reduced proliferation of tendon cells or whether there is an additional contribution of tendon cell migration.

Although carrier-treated mice restored gait and mechanical function after injury, gene expression analyses indicated a potential alteration or delay in healing at early timepoints. Our previous studies generally showed restored or upregulation of tendon markers by D14, however carrier-treated injured tendons in this study maintained decreased expression of Tnmd and Mkx. This may be due to an effect of the carrier solution on tendon healing. Similarly, II1 $\beta$ was also elevated in carrier-treated injured tendons at D14; in unpublished data from the lab using untreated mice, we generally observe resolution of neonatal inflammation at this timepoint (not shown). Interestingly, there was also an effect of AP20187-treatment on the contralateral uninjured tendon, in terms of tendon gene expression. While flow cytometry did not show a difference in macrophage number in control tendons with AP20187-treatment, we cannot rule out the possibility that there may be some degree of tendon-resident macrophage apoptosis leading to low-level local inflammation independent of injury. We also observed nearly complete macrophage depletion in the spleen, which may alter systemic circulating factors that may affect the contralateral control limb. In future studies, the impact of local vs systemic effects will be distinguished by local injection of AP20187 directly to the injured or control limb.

Finally, although macrophages are key players in the healing process, they are not the only immune cells that may regulate regeneration. During muscle regeneration, specific $\mathrm{T}$ cell subpopulations (Th1, Th2 and Tregs) have been shown to induce or impede regenerative healing (40-43). Our qPCR array screen also identified markers associated with $\mathrm{T}$ cells, consistent with previous studies showing their recruitment during tendon healing and in human tendinopathy (44-46). Ongoing work will test the role of T cells in tendon regeneration and scar formation.

\section{ACKNOWLEDGMENTS}

This work was supported by NIH/NIAMS (R01AR069537, R56AR076984) to AHH and F31AR073626 to DK. We gratefully thank Dr. Ronen Schweitzer for providing ScxGFP mice and the qPCR Core at Mount Sinai for their assistance with this project.

\section{CONFLICT OF INTEREST STATEMENT}

There are no conflicts of interest to disclose.

\section{AUTHOR CONTRIBUTIONS}

$\mathrm{KH}, \mathrm{DK}$, and $\mathrm{AHH}$ contributed to research conception and experimental design. $\mathrm{KH}, \mathrm{DK}, \mathrm{AM}$, $\mathrm{KY}$, and PN contributed to data collection. Data analyses were performed by $\mathrm{KH}, \mathrm{DK}$, and $\mathrm{AHH}$. Manuscript was written by $\mathrm{AHH}$ and all authors contributed to edits and approve this submission.

\section{REFERENCES}

1. Benjamin, M., Kaiser, E., and Milz, S. (2008) Structure-function relationships in tendons: a review. J Anat 212, 211-228 
2. Beason, D. P., Kuntz, A. F., Hsu, J. E., Miller, K. S., and Soslowsky, L. J. (2012) Development and evaluation of multiple tendon injury models in the mouse. $J$ Biomech 45, 1550-1553

\section{3. (2011) United States Bone and Joint Initiative: The Burden of Musculoskeletal Disease} in the United States, Second Edition, American Academy of Orthopaedic Surgeons, Rosemont, IL

4. Sereysky, J. B., Flatow, E. L., and Andarawis-Puri, N. (2013) Musculoskeletal regeneration and its implications for the treatment of tendinopathy. Int $J$ Exp Patho/ 94 , 293-303

5. Sharma, P., and Maffulli, N. (2005) Tendon injury and tendinopathy: healing and repair. J Bone Joint Surg Am 87, 187-202

6. Magnusson, S. P., and Kjaer, M. (2019) The impact of loading, unloading, ageing and injury on the human tendon. J Physiol 597, 1283-1298

7. Howell, K., Chien, C., Bell, R., Laudier, D., Tufa, S. F., Keene, D. R., Andarawis-Puri, N., and Huang, A. H. (2017) Novel Model of Tendon Regeneration Reveals Distinct Cell Mechanisms Underlying Regenerative and Fibrotic Tendon Healing. Sci Rep 7, 45238

8. Kaji, D. A., Howell, K. L., Balic, Z., Hubmacher, D., and Huang, A. H. (2020) TGFbeta signaling is required for tenocyte recruitment and functional neonatal tendon regeneration. eLife 9

9. Ackerman, J. E., Nichols, A. E., Studentsova, V., Best, K. T., Knapp, E., and Loiselle, A.

E. (2019) Cell non-autonomous functions of S100a4 drive fibrotic tendon healing. eLife 8

10. Zhang, K., Asai, S., Hast, M. W., Liu, M., Usami, Y., Iwamoto, M., Soslowsky, L. J., and Enomoto-Iwamoto, M. (2016) Tendon mineralization is progressive and associated with deterioration of tendon biomechanical properties, and requires BMP-Smad signaling in the mouse Achilles tendon injury model. Matrix Biol 52-54, 315-324

11. Arble, J. R., Lalley, A. L., Dyment, N. A., Joshi, P., Shin, D. G., Gooch, C., Grawe, B., Rowe, D., and Shearn, J. T. (2016) The LG/J Murine Strain Exhibits Near-Normal Tendon Biomechanical Properties Following a Full-Length Central Patellar Tendon Defect. Connect Tissue Res

12. Sunwoo, J. Y., Eliasberg, C. D., Carballo, C. B., and Rodeo. (2020) The role of the macrophage in tendinopathy and tendon healing. Journal of orthopaedic research : official publication of the Orthopaedic Research Society 38

13. Chisari, E., Rehak, L., Khan, W. S., and Maffulli, N. (2020) The role of the immune system in tendon healing: a systematic review. British medical bulletin 133

14. Kawamura, S., Ying, L., Kim, H. J., Dynybil, C., and Rodeo, S. A. (2005) Macrophages accumulate in the early phase of tendon-bone healing. J Orthop Res 23, 1425-1432

15. Sugg, K. B., Lubardic, J., Gumucio, J. P., and Mendias, C. L. (2014) Changes in macrophage phenotype and induction of epithelial-to-mesenchymal transition genes following acute Achilles tenotomy and repair. J Orthop Res 32, 944-951

16. Jomaa, G., Kwan, C. K., Fu, S. C., Ling, S. K., Chan, K. M., Yung, P. S., and Rolf, C. (2020) A systematic review of inflammatory cells and markers in human tendinopathy. BMC Musculoskelet Disord 21, 78

17. Chamberlain, C. S., Clements, A. E. B., Kink, J. A., Choi, U., Baer, G. S., Halanski, M. A., Hematti, P., and Vanderby. (2019) Extracellular Vesicle-Educated Macrophages Promote Early Achilles Tendon Healing. Stem cells (Dayton, Ohio) 37

18. Schoenenberger, A. D., Tempfer, H., Lehner, C., Egloff, J., Mauracher, M., Bird, A., Widmer, J., Maniura-Weber, K., Fucentese, S. F., Traweger, A., Silvan, U., and Snedeker, J. G. (2020) Macromechanics and polycaprolactone fiber organization drive macrophage polarization and regulate inflammatory activation of tendon in vitro and in vivo. Biomaterials 249 
19. Ackerman, J. E., Geary, M. B., Orner, C. A., Bawany, F., and Loiselle, A. E. (2017) Obesity/Type II diabetes alters macrophage polarization resulting in a fibrotic tendon healing response. PLoS One 12, e0181127

20. van Rooijen, N., and van Nieuwmegen, R. (1984) Elimination of phagocytic cells in the spleen after intravenous injection of liposome-encapsulated dichloromethylene diphosphonate. An enzyme-histochemical study. Cell and tissue research 238

21. de la Durantaye, M., Piette, A. B., van Rooijen, N., and Frenette, J. (2014) Macrophage depletion reduces cell proliferation and extracellular matrix accumulation but increases the ultimate tensile strength of injured Achilles tendons. J Orthop Res 32, 279-285

22. Godbout, C., Bilodeau, R., Van Rooijen, N., Bouchard, P., and Frenette, J. (2010) Transient neutropenia increases macrophage accumulation and cell proliferation but does not improve repair following intratendinous rupture of Achilles tendon. J Orthop Res 28, 1084-1091

23. Hays, P. L., Kawamura, S., Deng, X. H., Dagher, E., Mithoefer, K., Ying, L., and Rodeo, S. A. (2008) The role of macrophages in early healing of a tendon graft in a bone tunnel. $J$ Bone Joint Surg Am 90, 565-579

24. Best, K. T., Lee, F. K., Knapp, E., Awad, H. A., and Loiselle, A. E. (2019) Deletion of NFKB1 enhances canonical NF-kappaB signaling and increases macrophage and myofibroblast content during tendon healing. Sci Rep 9, 10926

25. Burnett, S. H., Kershen, E. J., Zhang, J., Zeng, L., Straley, S. C., Kaplan, A. M., and Cohen, D. A. (2004) Conditional macrophage ablation in transgenic mice expressing a Fas-based suicide gene. J Leukoc Biol 75, 612-623

26. Pryce, B., Brent, A., Murchison, N., Tabin, C., and Schweitzer, R. (2007) Generation of transgenic tendon reporters, ScxGFP and ScXAP, using regulatory elements of the scleraxis gene. Developmental dynamics : an official publication of the American Association of Anatomists 236, 1677-1682

27. Abraham, A. C., Shah, S. A., Golman, M., Song, L., Li, X., Kurtaliaj, I., Akbar, M., Millar, N. L., Abu-Amer, Y., Galatz, L. M., and Thomopoulos, S. (2019) Targeting the NFkappaB signaling pathway in chronic tendon disease. Sci Trans/ Med 11

28. Kurtaliaj, I., Golman, M., Abraham, A. C., and Thomopoulos, S. (2019) Biomechanical Testing of Murine Tendons. J Vis Exp

29. Wu, C. L., McNeill, J., Goon, K., Little, D., Kimmerling, K., Huebner, J., Kraus, V., and Guilak, F. (2017) Conditional Macrophage Depletion Increases Inflammation and Does Not Inhibit the Development of Osteoarthritis in Obese Macrophage Fas-Induced Apoptosis-Transgenic Mice. Arthritis \& rheumatology (Hoboken, N.J.) 69

30. Morris, M. W., Allukian, M., Herdrich, B. J., Caskey, R. C., Zgheib, C., Xu, J., DorsettMartin, W., Mitchell, M. E., and Liechty, K. W. (2014) Modulation of the inflammatory response by increasing fetal wound size or interleukin-10 overexpression determines wound phenotype and scar formation. Wound repair and regeneration : official publication of the Wound Healing Society [and] the European Tissue Repair Society 22

31. Walraven, M., Talhout, W., Beelen, R. H., van Egmond, M., and Ulrich, M. M. (2016) Healthy human second-trimester fetal skin is deficient in leukocytes and associated homing chemokines. Wound repair and regeneration : official publication of the Wound Healing Society [and] the European Tissue Repair Society $\mathbf{2 4}$

32. Herdrich, B. J., Danzer, E., Davey, M. G., Allukian, M., Englefield, V., Gorman, J. H., Gorman, R. C., and Liechty, K. W. (2010) Regenerative healing following foetal myocardial infarction. European journal of cardio-thoracic surgery : official journal of the European Association for Cardio-thoracic Surgery 38

33. Herdrich, B. J., Danzer, E., Davey, M. G., Bermudez, D. M., Radu, A., Zhang, L., Zhang, Z., Soslowsky, L. J., and Liechty, K. W. (2010) Fetal tendon wound size modulates wound gene expression and subsequent wound phenotype. Wound repair and 
regeneration : official publication of the Wound Healing Society [and] the European Tissue Repair Society 18

34. Longaker, M. T., Whitby, D. J., Ferguson, M. W., Lorenz, H. P., Harrison, M. R., and Adzick. (1994) Adult skin wounds in the fetal environment heal with scar formation. Annals of surgery 219

35. Favata, M., Beredjiklian, P., Zgonis, M., Beason, D., Crombleholme, T., Jawad, A., and Soslowsky, L. (2006) Regenerative properties of fetal sheep tendon are not adversely affected by transplantation into an adult environment. Journal of orthopaedic research : official publication of the Orthopaedic Research Society 24, 2124-2132

36. Debock, I., and Flamand, V. (2014) Unbalanced Neonatal CD4(+) T-Cell Immunity. Front Immunol 5, 393

37. Basha, S., Surendran, N., and Pichichero, M. (2014) Immune responses in neonates. Expert Rev Clin Immunol 10, 1171-1184

38. Beredjiklian, P., Favata, M., Cartmell, J., Flanagan, C., Crombleholme, T., and Soslowsky, L. (2003) Regenerative versus reparative healing in tendon: a study of biomechanical and histological properties in fetal sheep. Annals of biomedical engineering 31, 1143-1152

39. Blomgran, P., Hammerman, M., and Aspenberg, P. (2017) Systemic corticosteroids improve tendon healing when given after the early inflammatory phase. Scientific reports 7

40. Burzyn, D., Kuswanto, W., Kolodin, D., Shadrach, J. L., Cerletti, M., Jang, Y., Sefik, E., Tan, T. G., Wagers, A. J., Benoist, C., and Mathis, D. (2013) A special population of regulatory $T$ cells potentiates muscle repair. Cell 155, 1282-1295

41. Kuswanto, W., Burzyn, D., Panduro, M., Wang, K. K., Jang, Y. C., Wagers, A. J., Benoist, C., and Mathis, D. (2016) Poor Repair of Skeletal Muscle in Aging Mice Reflects a Defect in Local, Interleukin-33-Dependent Accumulation of Regulatory T Cells. Immunity 44, 355-367

42. Sadtler, K., Estrellas, K., Allen, B. W., Wolf, M. T., Fan, H., Tam, A. J., Patel, C. H., Luber, B. S., Wang, H., Wagner, K. R., Powell, J. D., Housseau, F., Pardoll, D. M., and Elisseeff, J. H. (2016) Developing a pro-regenerative biomaterial scaffold microenvironment requires T helper 2 cells. Science 352, 366-370

43. Ito, M., Komai, K., Mise-Omata, S., lizuka-Koga, M., Noguchi, Y., Kondo, T., Sakai, R., Matsuo, K., Nakayama, T., Yoshie, O., Nakatsukasa, H., Chikuma, S., Shichita, T., and Yoshimura, A. (2019) Brain regulatory T cells suppress astrogliosis and potentiate neurological recovery. Nature 565, 246-250

44. Blomgran, P., Blomgran, R., Ernerudh, J., and Aspenberg, P. (2016) A possible link between loading, inflammation and healing: Immune cell populations during tendon healing in the rat. Scientific reports 6

45. Millar, N. L., Akbar, M., Campbell, A. L., Reilly, J. H., Kerr, S. C., McLean, M., FrletaGilchrist, M., Fazzi, U. G., Leach, W. J., Rooney, B. P., Crowe, L. A., Murrell, G. A., and Mclnnes, I. B. (2016) IL-17A mediates inflammatory and tissue remodelling events in early human tendinopathy. Sci Rep 6, 27149

46. Noah, A. C., Li, T. M., Martinez, L. M., Wada, S., Swanson, J. B., Disser, N. P., Sugg, K. B., Rodeo, S. A., Lu, T. T., and Mendias, C. L. (2020) Adaptive and Innate Immune Cell Responses in Tendons and Lymph Nodes After Tendon Injury and Repair. Journal of applied physiology 